\title{
First report of sandfly fever virus infection imported from Malta into Switzerland, October 2011
}

D Schultze (detlev.schultze@zlmsg.ch) ${ }^{1}$, W Korte ${ }^{1}$, P Rafeiner², M Niedrig ${ }^{3}$

1. Center of Laboratory Medicine, St. Gallen, Switzerland

2. Department of Internal Medicine, Division of Infectious Diseases, Cantonal Hospital, St Gallen, Switzerland

3. Center for Biological Security (ZBS-1), Robert Koch Institute, Berlin, Germany

Citation style for this article:

Schultze D, Korte W, Rafeiner P, Niedrig M. First report of sandfly fever virus infection imported from Malta into Switzerland, October 2011. Euro Surveill. 2012;17(27):pii=20209. Available online: http://www.eurosurveillance.org/ViewArticle.aspx?Articleld=20209

Article submitted on 22 June 2012 / published on 5 July 2012

We report the first documented cases of sandfly fever virus infection in travellers returning from Malta to Switzerland in autumn 2011. These cases illustrate the importance of considering sandfly-borne viral infection in the differential diagnosis of febrile patients from the Mediterranean island Malta. Raising awareness among physicians is relevant especially now at the beginning of the summer tourist season.

On 17 October 2011, a Swiss citizen was hospitalised with fever, nausea, vomiting and intensifying headache two days after his return from the Mediterranean island Malta, where he had spent two weeks on Gozo Island. Nine days before his admission, the patient had suffered from back pain, tiredness and subfebrile temperatures, but had recovered after medication with the non-steroid inflammatory drug ibuprofen. The patient's wife had suffered from similar, less intense symptoms. Both had multiple insect bites and were diagnosed with laboratory-confirmed sandfly fever. The couple's two accompanying children and two further travel companions had been frequently bitten by small flying insects, but suffered no symptoms.

\section{Case 1}

Case 1, a man in his late 40s, was admitted to hospital in Switzerland two days after his return from Malta and presented in a reduced general condition. He had fever $\left(38.4^{\circ} \mathrm{C}\right)$, a generalised rash and complained of severe headache, without meningism. Multiple skin lesions due to insect bites were visible on the extremities (Figure).

Laboratory tests for this patient, including C-reactive protein, electrolytes and transaminases revealed no abnormalities, and a complete blood cell count showed relative lymphopenia (17.9\%, normal range: $20-52 \%)$.

Because of the recent stay in a Mediterranean country and the multiple skin lesions, a sandfly fever virus (SFV) infection (pappataci fever) was assumed. Serology for SFV was positive on a serum sample taken on the day after admission, i.e. 10 days after onset of first symptoms on Malta. An immunoblot (IB) for bunyaviruses (Mikrogen, Munich, Germany) showed reactivity with the TOSV bands, with stronger intensity than the cut-off reference, indicating the presence of $\operatorname{lgM}$ and IgG antibodies against TOSV. An indirect immunofluorescence test (IIFT) (Sandfly fever virus Mosaic 1, Sandfly fever virus serotypes Sicilian, Naples, Toscana, Cyprus; Euroimmun, Lübeck, Germany) revealed high serum antibody titres against Toscana and Naples SFV (Table), indicating an infection with a phlebovirus belonging to the SFNV serological complex.

In a convalescent serum, taken 52 days after onset of illness, an eight-fold increase of anti-TOSV IgM was demonstrated, accompanied by a five-fold increase for anti-TOSV IgG (Table).

The patient gradually improved under analgesic and anti-emetic therapy. On day 15 after onset of illness he could be transferred to a rehabilitation centre and fully

\section{FIGURE}

Multiple skin lesions due to the insect bites on arm of sandfly fever patient, Switzerland, October 2011

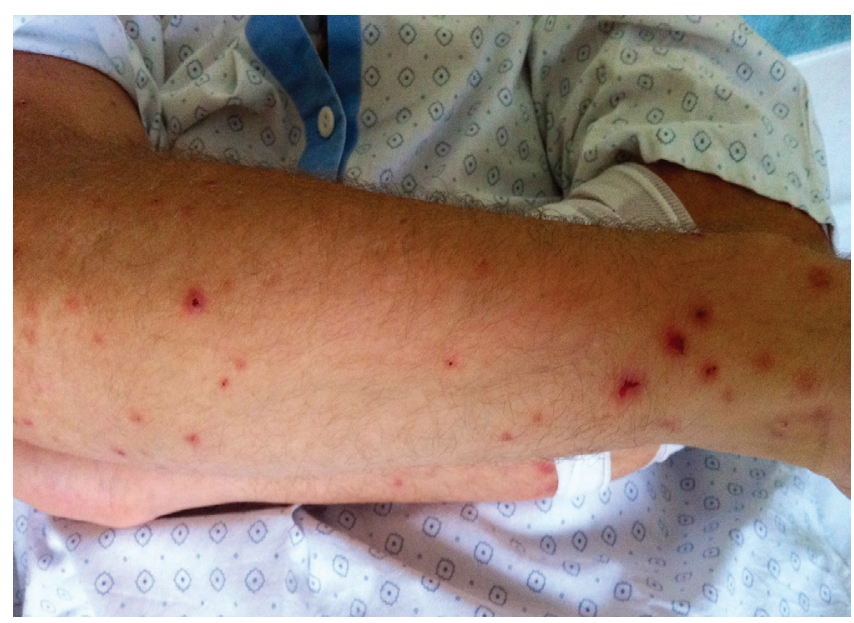


Serology results for two patients with sandfly fever, Switzerland ex Malta, October 2011

\begin{tabular}{|l|c|c|c|c|}
\hline & \multicolumn{2}{|c|}{ Case 1} & \multicolumn{2}{c|}{ Case } \\
\hline Days post-onset of illnesS & 10 & 52 & +1 & + - \\
\hline TOSV IgM IB & ++ & ++ & ++ \\
\hline TOSV IgG IB & ++ &,++ 200 & 100 & 1,000 \\
\hline TOSV IgM IIFT & 400 & 10,000 & - & 1,000 \\
\hline TOSV IgG IIFT & 2,000 & 1,000 & - & 320 \\
\hline SFNV IgM IIFT & 400 & 1,000 & - & 100 \\
\hline SFNV IgG IIFT & 1,000 & - & - & - \\
\hline SFSV IgM IIFT & - & - & - & - \\
\hline SFSV IgG IIFT & - & - & - & - \\
\hline SFCV IgM IIFT & - & - & & - \\
\hline SFCV IgG IIFT & - & & - \\
\hline
\end{tabular}

IB: bunyavirus immunoblot, IIFT: indirect immunofluorescence test; SFCV: sandfly fever Cyprus virus; SFNV: sandfly fever Naples virus; SFSV: sandfly fever Sicilian virus; TOSV: Toscana virus.

++ strong reactivity,

+ - weak reactivity,

no reactivity/no immunofluorescence.

Numbers refer to reciprocal titres, according to the dilution scheme of the manufacturer. End-point titres were adjusted to the strength of immunofluorescence, e.g. end-point titre 1:3,200, if serum-dilution 1:1,000 showed medium strength of immunofluorescence.

recovered 21 days after onset of symptoms. The patient suffered no relapse in the following eight months.

\section{Case 2 and accompanying persons}

Case 2, a woman in her early 50s, showed positive IgM antibodies against TOSV and SFNV in a serum taken 11 days after onset of illness, with a 10 -fold increase in anti-TOSV IgM and a seroconversion for anti-TOSV IgG antibodies in a follow-up serum, taken on day 52 after onset of illness (Table).

Serum samples were also taken from the couple's children and the two accompanying travellers on day 52 after onset of illness of patients 1 and 2. All samples were tested by IIFT and IB and turned out negative for IgM and IgG antibodies against TOSV, SFNV, SFSV, and SFCV.

Leishmania species are known to be transmitted by Phlebotomus perniciosus in Malta [5]. Patients were informed to remain alert for late appearing symptoms of leishmaniasis, which may not manifest until months after infection [5]. However, in the following eight months, none of the travellers developed clinical signs of leishmaniasis.

\section{Background}

Phleboviruses of the Bunyaviridae family, transmitted to humans by arthropods, are found in Europe, Africa, central Asia, and the Americas. In Mediterranean Europe, the phleboviruses Toscana virus (TOSV), sandfly fever Naples virus (SFNV), sandfly fever Sicilian virus (SFSV), and sandfly fever Cyprus virus (SFCV) are transmitted by phlebotomine sandflies. Among these, TOSV circulates in countries around the Mediterranean Sea (Algeria, Cyprus, France, Greece, Italy, Portugal, Spain and Turkey) $[1,2]$.

TOSV may cause an acute, nonfatal, influenza-like symptomatology or even aseptic meningitis and meningoencephalitis [2-4]. SFNV, SFSV and other related viruses can cause the so-called 'three-day fever' or 'pappataci fever'. Patients present with influenza-like symptoms including fever, retro-orbital pain, myalgia and malaise and usually recover fully within a week. However, infections with these viruses, even when mild, have been shown to be highly incapacitating during the time the patients are affected [2].

\section{Discussion}

Viruses in the genus Phlebovirus can cause a variety of clinical syndromes ranging from a brief, self-limiting febrile illness to encephalitis, meningoencephalitis and fatal haemorrhagic fever. Of our cases, the male patient suffered from symptoms of a SFV infection that incapacitated him for two weeks, while his wife had less severe symptoms. The other four travellers accompanying the couple were not infected, despite multiple insect bites.

The Phlebovirus genus consists of more than 60 distinct virus serotypes. While antigenically unrelated to members of other genera in the family Bunyaviridae, various degrees of cross-reactivity in serological tests can occur within the genus, as reported for the SFNV and SFSV antigenic complexes [6,7]. Our patients showed 
cross-reactions in the IIFT using SFNV and TOSV antigens, both members of the SFNV antigenic complex. Although IIFT using SFSV and SFCV as antigens yielded negative results, reactions with other serotypes in the genus Phlebovirus cannot be ruled out.

Viral neutralisation tests (VNT) using early convalescent sera remain the serological reference method to identify these viruses or to assess the specificity of the antibody response [8]. Although generally regarded as the gold standard assay for specificity, the VNT is relatively labour-intensive and only established in few laboratories. SFV-specific commercial assays such as IIFT, enzyme-linked immunoassay and IB are much more commonly used. For the diagnosis of the cases described here, we used IIFT for screening purposes and the IB as confirmatory assay [8].

Direct viral diagnosis by isolation and RT-PCR from blood or cerebrospinal fluid is only possible in the early stages of infection, i.e. the first two days after onset of symptoms and before seroconversion [2]. Case 1 presented on day 9 after symptom onset, at a time when SFV-specific serology was already positive. Diagnosis was therefore attempted by serological investigation, using two commercial kits. That the increase in antiTOSV IgM and IgG in the convalescent serum of Case 1 was higher than the increase in antibodies against SFNV, was suggestive of an infection with TOSV rather than with SFNV. Similarly in Case 2, anti-TOSV IgM increased more than anti-SFNV IgM and was accompanied by higher seroconversion for anti-TOSV IgG than for SFNV-IgG.

Most cases of TOSV infection have been reported in residents of or travellers to central Italy and Spain, and sporadically in other Mediterranean regions such as Portugal, Cyprus, southern France and Greece [9]. Asymptomatic infections have also been described [9]. Unlike the other SFV serotypes, TOSV shows a peculiar neurotropism. It can cause meningitis or meningoencephalitis from which patients generally recover within seven to 10 days [9]. TOSV infections occur particularly during the summer and correlate with the life cycle of the insect vectors Phlebotomus perniciosus and P. perfiliewi [9].

TOSV has been isolated from $P$. perfiliewi and $P$. perniciosus. The latter vector is distributed throughout the Mediterranean region as two races. SFNV has been isolated in Italy from $P$. perniciosus, in Serbia from $P$. perfiliewi and in Egypt from $P$. papatasi [10]. The typical $P$. perniciosus race occurs in Italy as well as in Tunisia, Morocco and in Malta [10]. However, to the best of our knowledge, neither TOSV nor SFNV infections have been reported from Malta so far. Thus, in connection with the beginning of the summer season, it is now of particular relevance to consider sandflyborne phleboviruses in the differential diagnosis of patients returning from Malta and presenting with febrile illness.

\section{References}

1. Ergunay K, Aydogan S, Ilhami Ozcebe O, Cilek EE, Hacioglu S Karakaya J, et al. Toscana virus (TOSV) exposure is confirmed in blood donors from Central, North and South/Southeast Anatolia, Turkey. Zoonoses Public Health. 2012;59(2):148-54. doi: $10.1111 / j .1863-2378.2011 .01436 . x$.

2. Depaquit J, Grandadam M, Fouque F, Andry P, Peyrefitte C. Arthropod-borne viruses transmitted by Phlebotomine sandflies in Europe: a review. Euro Surveill. 2010;15(10): pii=19507. Available from: http://www. eurosurveillance.org/ViewArticle.aspx?Articleld $=19507$

3. Terrosi C, Olivieri R, Bianco C, Cellesi C, Cusi MG. AgeDependent Seroprevalence of Toscana Virus in Central Italy and Correlation with the Clinical Profile. Clin Vaccine Immunol. 2009;16(8):1251-2.

4. Dionisio D, Esperti F, Vivarelli A, Valassina M. Epidemiological, clinical and laboratory aspects of sandfly fever. Curr Opin Infect Dis. 2003;16(5):383-8.

5. Pace D, Williams TN, Grochowska A, Betts A, Attard-Montalto S, Boffa MJ, et al. Manifestations of paediatric Leishmania infantum infections in Malta. Travel Med Infect Dis. 2011;9(1):37-46.

6. Liu DY, Tesh RB, Travassos Da Rosa AP, Peters CJ, Yang Z, Guzman $\mathrm{H}$, et al. Phylogenetic relationships among members of the genus Phlebovirus (Bunyaviridae) based on partial M segment sequence analyses. J Gen Virol. 2003;84(Pt2):465-73.

7. Xu F, Chen H, Travassos da Rosa AP, Tesh RB, Xiao SY. Phylogenetic relationships among sandfly fever group viruses (Phlebovirus: Bunyaviridae) based on the small genome segment. J Gen Virol. 2007; 88(Pt2): 2312-9.

8. Ergunay K, Litzba N, Lo MM, Aydoğan S, Saygan MB, Us D, et al. Performance of various commercial assays for the detection of Toscana virus antibodies. Vector Borne Zoonotic Dis. 2011;11(6):781-7

9. Baldelli F, Ciufolini MG, Francisci D, Marchi A, Venturi G, Fiorentini C, et al. Unusual Presentation of Life-Threatening Toscana Virus Meningoencephalitis. Clin Infect Dis. 2004;38(4):515-20.

10. Charrel RN, Gallian P, Navarro-Mari JM, Nicoletti L, Papa A, Sánchez-Seco MP, et al. Emergence of Toscana Virus in Europe. Emerg Infect Dis. 2005;11(11):1657-63. 